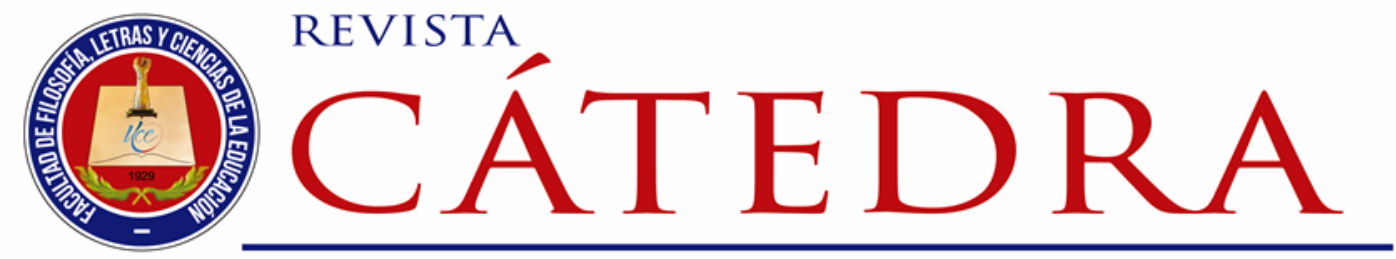

\title{
Los roles de género en el concepto de familia fang: un sistema de socialización diferencial
}

\section{Gender roles in the fang family concept: a differential socialization system}

\author{
Pedro Bituga-Nchama \\ Universidad Nacional de Guinea Ecuatorial, Bata, Guinea Ecuatorial \\ pedrobayem@gmail.com \\ https://orcid.org/0000-0003-2310-5879
}

(Recibido: 25/02/2021; Aceptado: 1/03/2021; Versión final recibida: 15/04/2021)

Cita del artículo: Bituga-Nchama, P. (2021). Los roles de género en el concepto de familia fang: un sistema de socialización diferencial. Revista Cátedra, 4(2), 88-105.

\section{Resumen}

Este artículo se centra en visibilizar los roles de género, productos de la socialización diferencial del concepto de familia fang. Este objetivo fundamental pone de manifiesto que al ser la familia una institución en la que se produce la primera socialización de las personas, el modelo de familia fang no se aleja de este estándar, porque la manera de socializar dentro de esta cultura, hace que a los hombres se les asignen unos roles diferentes a los de las mujeres. Esto se ve notablemente en la división sexual del trabajo que está muy presente en el concepto de familia fang, perpetuando así el sistema de socialización diferencial que impulsa y agrava las desigualdades sociales, motivadas por el sexo-género. En este sentido, encontramos que las tareas domésticas y el espacio privado, corresponden exclusivamente a las mujeres, mientras que los hombres ocupan el espacio público. Una sociedad regida por este sistema de socialización diferencial crea un desequilibro donde las mujeres terminan siendo minimizadas. A fin de estudiar mejor esta temática, se ha utilizado la metodología cuantitativa, partiendo del método descriptivo, el cual ha permitido reflejar la incidencia de este fenómeno en el grupo poblacional seleccionado. En esta investigación se reafirma que, los roles de género tradicionales se promueven de distintas maneras dentro del concepto de familia fang fomentando una educación de dependencia, donde las aptitudes cognitivas y personales de las mujeres son siempre infravaloradas por el sistema patriarcal fang. 


\section{Palabras clave}

Familia, género, patriarcado, rol, socialización.

\section{Abstract}

This article focuses on making visible the gender roles, products of the differential socialization of the concept of the Fang family. This fundamental objective shows that since the family is an institution where the first socialization of people takes place, the Fang family model is not far from this standard, because the way of socialization within this culture makes men be assigned different roles than women. This is notably seen in the sexual division of labor that is very present in the concept of the Fang family, thus perpetuating the differential socialization system that drives and aggravates social inequalities, motivated by sex-gender. In this sense, we find that domestic tasks and private space correspond exclusively to women, while men occupy public space. A society governed by this differential socialization system creates an imbalance where women end up being minimized. In order to better study this issue, a quantitative methodology has been used, based on the descriptive method, which has made it possible to reflect the incidence of this phenomenon in the selected population group. This research reaffirms that traditional gender roles are promoted in different ways within the concept of the Fang family, fostering an education of dependence, where the cognitive and personal skills of women are always undervalued by the Fang patriarchal system.

\section{Keywords}

Family, gender, patriarchy, role, socialization.

\section{Introducción}

El presente artículo tiene por objeto analizar los roles de género en el concepto de familia fang desde la socialización diferencial de la niñez. En este sentido, se pretende poner de manifiesto el sistema de socialización diferencial y complejo que se lleva a cabo a la hora de determinar lo masculino y lo femenino. Un hecho real es que por el modelo de familia fang, al igual que en otros pueblos africanos, la educación de la niñez no depende exclusivamente de los progenitores, sino que de toda la comunidad.

Teniendo en cuenta el impacto de la socialización diferencial en la educación dentro del concepto de familia fang, como el principal agente de socialización donde se construye lo masculino y lo femenino, en este estudio se analiza este fenómeno partiendo de la literatura existente sobre el particular.

Una de las instituciones respetadas por los fang es la familia, por eso, aquella no se agota exclusivamente con el padre o la madre, pues va más allá, porque abarca incluso a gente que no tiene ningún lazo sanguíneo en común, sino por el parentesco de un antepasado común o un tótem. Para los fang, la institución familiar es muy importante ya que es el centro en el que se forma a la persona humana. La familia en la comunidad fang sigue la misma tendencia que la de otros pueblos de África. Así pues, se ha encontrado que:

Para los pueblos africanos, la familia consiste en un círculo de personas mucho más amplio que en el mundo occidental. En la comunidad tradicional, la familia incluye a los hijos, padres, abuelos, tíos, tías, hermanos, hermanas que, a su vez, tienen sus propios hijos, además de otros parientes inmediatos (Mbiti, 1990, pág. 142). 
Esta cuestión de la que habla el autor citado, presenta la idea de que en África la familia se extiende mucho más allá del círculo paterno. La familia constituye uno de los grandes valores que poseen los pueblos africanos y sobre ella reposa fundamentalmente la sociedad. Los lazos de parentesco que se establecen en el pueblo fang condicionan a los individuos que pertenecen a esta tribu o familia. Su manera de actuar, hablar o reaccionar no es igual para los que no son de su tribu. En este sentido, el concepto de familia fang sigue siendo sólido y los lazos de sangre constituyen un nexo irrompible. Si bien es cierto que en todos los pueblos de África en general y en particular los de Guinea Ecuatorial, la estructura familiar no es nuclear, hay que destacar que, en la actualidad, dicha estructura se ha deteriorado en ciertos individuos modernizados e influenciados por la globalización, lo que ha hecho que se desdibuje el concepto de familia que anteriormente tenía la comunidad fang. No obstante, a pesar de los múltiples cambios, "la familia africana hoy como en el pasado, se extiende desde el núcleo restringido (padres-hijos) hasta todo el engranaje del parentesco donde las costumbres y los lazos tradicionales se conjugan para formar un sistema conexo e indisoluble" (Ki-Zerbo, 1972, pág. 22).

La socialización diferencial dentro del concepto de familia fang es el resultado del sistema patriarcal muy consolidado sobre el que se sustenta esta etnia, de tal forma que la trasmisión de los roles de género se hace desde la perspectiva androcéntrica donde las niñas terminan siendo educadas para estar al servicio de los niños. Los roles de género de los que se hablan aquí, no son un hecho fortuito, sino que son los que sustentan la subordinación de la mujer a la que se promueve con la socialización diferencial del sistema patriarcal. Niñas y niños son socializados desde temprano para comportarse de una determinada manera, tal y como lo quieren las personas adultas que les están educando. Por ello, problemas como la violencia de género, la poligamia, el levirato, los abortos, la educación, son cuestiones de actualidad en la sociedad ecuatoguineana, pero son abordados y son resueltos desde una postura patriarcal, sin considerar la perspectiva de género.

Por tanto, el problema que se plantea en esta investigación tiene que ver con la manera en que, desde la socialización diferencial, se educa a los niños y niñas dentro del modelo familiar fang, asignando a las niñas unos valores y a los niños otros. Lo que se pretende con este modelo de educación es establecer claramente el espacio que debe ocupar cada uno, y los roles que debe desempeñar a nivel social, de esta manera, es evidente que las niñas son educadas para ser madres, casarse y cuidar a sus esposos e hijos. Los roles de género asignados son el resultado de la manera en que se quiere que sea una mujer fang, es decir, sumisa, mientras que el hombre es dominante.

En relación a lo anterior, el problema planteado se asienta sobre unas cuestiones que son claves para desentrañar mejor la temática que se está abordando. Estas preguntas de investigación a las que se pretender dar respuesta son las siguientes: ¿En qué consiste el concepto de familia fang? ¿Cómo influye la socialización diferencial en el concepto de familia fang? ¿Hasta qué punto los roles de género agravan la subordinación de la mujer en la cultura fang? ¿De qué manera la socialización diferencial ayuda a mantener el sistema patriarcal fang?

La asimetría que se puede entrever dentro del concepto de familia fang en relación a la trasmisión de los roles de género desde la etapa más temprana, permite a los niños y niñas identificarse con ciertos valores y pautas de comportamiento. En el caso de los niños estos son moldeados para dominar mientras las niñas son educadas para ser pasivas. En suma, la socialización diferencial permite de igual modo que los niños reciban una educación diferente al de las niñas, algo que después es reforzado en la escuela. 
Si la asignación de roles de género es producto de la socialización diferencial en la que los hombres son educados como seres dominantes y las mujeres como sumisas. Esta forma de educar tiene relación con la visión androcentrista. En este sentido, la tesis que se defiende en este trabajo de investigación es la siguiente: el concepto de familia fang promueve la socialización diferencial a través de la transmisión de los roles de género que convierten a la mujer en una sumisa, robusteciendo el sistema patriarcal sobre el que se sustenta este modelo de socialización.

Este artículo se encuentra estructurado principalmente en tres partes. La primera corresponde al estado de la cuestión, que es la fundamentación teórica en la que se aborda la terminología de los roles de género y la socialización diferencial. En la segunda parte, se recoge la defensa de la tesis, donde se presenta una serie de argumentos que evidencian la socialización diferencial en el concepto de familia fang. La última parte, es la exposición de las conclusiones a las que se ha llegado con esta investigación.

\section{Estado de la cuestión}

La literatura existente sobre la socialización diferencial y los roles de género es amplísima. No obstante, en el contexto africano se encuentra una serie de problemas a la hora de abordar estos conceptos, debido principalmente a la escasez de fuentes bibliográficas sobre este tema. A lo largo de los años, tanto la sociología, antropología o la etnografía, se han ocupado de estudiar el concepto de familia africana, comparándolo a veces con otros modelos de familia como la occidental. Sin embargo, no se ha mostrado el mismo interés académico sobre los roles de género dentro de las sociedades africanas en general.

Debido al modelo de socialización diferencial, existe como se ha mencionado anteriormente, una manera concreta de educar a los niños y niñas. Todo ello, es lo que hace que los niños sean socializados de manera diferente a las niñas, las cuales terminan aprendiendo unos valores, creencias o pautas de comportamiento establecidos para servir al hombre. Antes de entrar profundamente en este tema, conviene sentar las bases teóricas sobre términos claves para entender mejor esta cuestión. En este sentido, es conveniente ofrecer algunas aproximaciones conceptuales sobre la familia, la socialización diferencial y los roles de género, para así poder estudiar el concepto de familia fang.

La familia es una de las primeras instituciones en la socialización de las personas. Por ello, es importante atender a que, en la familia las personas terminan asimilando desde la edad temprana, todo aquello que les dice o enseña. La socialización es determinada por la familia, la cual impone o decide sobre cómo educar a sus hijos e hijas.

Aun cuando existan diferentes modelos de familia en función de cada cultura, hay un hecho común a todas ellas, el cual está en que "la familia es el primer contexto donde comenzamos nuestro desarrollo, donde establecemos nuestras primeras relaciones sociales con diferentes personas y comenzamos a desarrollar una imagen de nosotros mismos y del mundo que nos rodea" (Musitu \& Cava, 2001, pág. 11). Por tanto, se trata de un núcleo fundamental para construir la identidad de las personas partiendo de su sexo.

Se aprende a ser niños o niñas, hombres o mujeres, partiendo de las expectativas de la gente que tuvo que socializarnos dentro de nuestra familia. Téngase en cuenta que no se trata de una educación común a todas las familias, debido fundamentalmente porque "Cada familia es un mundo y tiene sus características individuales que la hacen diferente de otra" (Meil Landwerlin, 2006, pág. 105). Todo ello, hace que se eduque de manera diferente de acuerdo al tipo de familia que se trate, aunque en última instancia, el objetivo fundamental de esta educación es socializar a la gente. 
Las recetas de cómo se debe educar a las personas están predichas por la cultura, la cual se encarga de facilitar a las familias unas herramientas necesarias para que aquellos que van a formar parte de la sociedad, actúan en base a unos cánones. Las expectativas de los progenitores son muy importantes a la hora de establecer la socialización diferencial, asignando unos roles concretos para los hombres y otros para las mujeres. El proceso de socialización diferencial que se inicia con la familia, se ve reforzado por la escuela, donde el niño o la niña puede darse cuenta que les exigen ciertas cosas por su sexo, las cuales deben hacer porque socioculturalmente es aquello que les identifica.

Por esta razón, se define la socialización como "un proceso por medio del cual los niños desarrollan hábitos, habilidades, valores y motivos que los hacen miembros responsables y productivos en la sociedad" (Papalia, 2005, pág. 231). Por consiguiente, se trata de un proceso vinculado a la educación, puesto que es el momento en el que el niño aprende el lenguaje, la conducta, la cultura, etc. Comienza de igual forma a ver cómo el padre realiza ciertas funciones que la madre no realiza. Uno de los aspectos importantes de esta primera fase de socialización es que el niño o la niña aprende a identificarse con su sexo, en base a los roles que debe ejercer. En este sentido, la socialización se define como:

El proceso mediante el que las personas adquirimos los valores, las creencias, las normas, y las formas de conducta apropiados en la sociedad a la que pertenecemos. A esta definición podríamos añadirle el concepto de interacción social, ya que a través de esta es como aprendemos y reproducimos los patrones culturales, sociales, religiosos, de género, etc. (Musitu \& Cava, 2001, pág. 115).

Tal como apuntan los investigadores mencionados en la cita anterior, la socialización tiene que ver con la manera en la que adquirimos toda la información que necesitamos para nosotros, y que a lo largo de nuestra vida esta información se va perfeccionando. Por ello, la familia no deja de jugar un rol importante en la socialización porque es el momento crucial donde se construyen las identidades, donde niños y niñas asumen roles diferentes. Según algunos investigadores, "el agente socializador por excelencia es la familia" (MartínezGonzález, 1996, pág. 88), porque es el núcleo donde los individuos asimilan los valores, creencias y pautas de comportamientos que les inculcan las personas adultas de su comunidad.

La familia es el germen donde se produce una socialización diferencial, por tanto, una construcción de lo masculino y femenino. Los roles de género ocupan un lugar tan preponderante para la construcción de las identidades. Y eso, no depende necesariamente de si se trata de un modelo de familia occidental, africana o asiática, en todos ellos hay un componente común, y es que, la gente es socializada de tal manera que cada uno funcione de acuerdo las pautas de comportamiento que se le asigna socialmente. La gran influencia que ejerce la familia a la hora de socializar de manera diferenciada a los individuos, se plantea argumentando que:

La familia se constituye en el vehículo transmisor de pautas culturales a través de varias generaciones permitiendo al mismo tiempo modificaciones de las mismas. Entre sus objetivos se encuentran; la protección y continuación de la crianza; La enseñanza del comportamiento e interacción con la sociedad; la adquisición de una identidad de género; la inculcación de valores sociales, éticos, morales; la conformación de una identidad personal, familiar y social (Ares-Muzio, 2002, págs. 18-19). 
La transmisión de los roles de género por parte de la familia viene a ser de esta manera, un componente de la socialización en el que aprendemos a funcionar de una determinada manera. Esta manera de construir identidades a partir de la socialización, no es ajena al concepto de familia fang, donde aun cuando la socialización primaria no es ejercida únicamente por los progenitores, termina por determinar cómo van a ser estos individuos.

El problema que subyace en lo anteriormente planteado, y que se va a desarrollar a continuación, tiene que ver con la socialización diferencial, que refuerza las desigualdades sociales y agrava la subordinación femenina en la cultura fang.

\section{Defensa de la tesis}

Tras sentar las bases teóricas de esta investigación, es conveniente recordar que la tesis que se defiende es la siguiente: el concepto de familia fang promueve la socialización diferencial a través de la transmisión de los roles de género que convierten a la mujer en una sumisa, robusteciendo el sistema patriarcal sobre el que se sustenta este modelo de socialización. Cuando se habla de roles de género, es necesario remarcar que "son aquellas normas sociales y comportamentales establecidas y percibidas como propias del hombre y de la mujer en función de la construcción social que se tiene de la masculinidad y feminidad en sociedad" (Myers, 2006, pág. 980).

Partiendo de este planteamiento, es prudente señalar que la socialización diferencial marca en gran medida los roles de género dentro del concepto de familia fang. Si bien la categoría de género no es la única para establecer una socialización diferencial, determina el hecho de que las mujeres pasen a ser dominadas por los hombres. En este sentido, la familia constituye una de las grandes instituciones que poseen los africanos y sobre ella reposa fundamentalmente la sociedad. En función de lo anterior, siguiente argumento es decisivo por señalar que:

La realidad muestra que llegar a entender antropológicamente la familia aparece como requisito para plantearse seriamente acerca de su impacto sobre el desarrollo humano armónico, el crecimiento de la sociedad que se vincula necesariamente con esta realidad humana (Santelices-Cuevas, 2001, págs. 183-198).

Por esta razón resulta imperioso entender los roles de género en el concepto de familia fang, como parte de la gran familia africana. Tal es así que:

Hablar de la familia africana tradicional es hablar de una familia amplia. Esta se define como el conjunto de personas procedentes de un antepasado común vivo, reunidas en un mismo lugar -generalmente la concesión-, sometidas al mismo jefe -el de más edad de la generación más antigua (padre o tío uterino)-, responsable de la vida económica -él es gerente de los bienes colectivos-y del culto dado a los poderes telúricos y a los espíritus ancestrales de los que él es sacerdote (Maurier, 1966, pág. 195).

Con ello, la socialización no depende solo de los progenitores, sino que se extiende más allá de este círculo. La educación de los niños y niñas es llevada a cabo por toda la comunidad donde cada uno va identificándose con aquello que debe realizar en función de su sexo. Los fang, como parte de la cultura africana, no se alejan de la realidad que se puede observar en los pueblos africanos. Sobre el valor de la familia en la etnia fang, algunos autores señalan 
un dato que parece importante destacar, porque recoge detalladamente el sentido de la familia para los fang.

La familia fán tiende a ser más amplia y ofrece una estructura compleja; la extensión del parentesco en las normas de interacción social suele ser mucho mayor. Esto proviene la inclusión de la familia primaria en agrupaciones de parentesco más vasto. La familia fán constituye un grupo social distinto de la familia europea; y las relaciones de parentesco son concebidas de un modo más diverso que en Europa (Nzé-Abuy, 1985, pág. 9).

El sistema patriarcal fang está fraguado para que el hombre cuide y proteja a la mujer, por considerarla inferior en muchos aspectos, como la capacidad de raciocinio, la gestión de los recursos económicos del hogar, etc. Como ha ocurrido en otras partes, el patriarcado es la manifestación de la autoridad feroz del hombre sobre la mujer.

Este gobierno patriarcal que, en principio, se ha vivido y se vive en otras sociedades, según algunos postulados feministas, "se caracteriza por la autoridad de los hombres sobre las mujeres y sus hijos impuesta desde las instituciones" (Castells, 1998 , pág. 159). En este sentido, la institución de los fang que ha impuesto esta autoridad de los hombres es la familia. De hecho, en el sistema familiar fang que se ha descrito ut supra, el hombre tiene un rol activo ya que es quien provee a la mujer de lo que necesita para la supervivencia.

El sistema patriarcal fang es el resultado de la autoridad de los hombres sobre la vida en la sociedad. Así pues, vemos que "la orientación familiar fang, ha sido, siempre y hasta hoy, patrilineal o de la línea del padre [...], los hijos se suceden y siguen la línea del padre" (Mibui, 2005, págs. 91-92). Al estar constituido de esta manera la orientación familiar Fang, los hombres se convierten en adalides de sus comunidades o aldeas, en las que arbitrariamente deciden desde la perspectiva androcéntrica, la manera en la que debe funcionar cada uno de los individuos que forma parte de estas comunidades.

La educación es un factor muy importante que ha ayudado a mantener la sumisión de la mujer Fang. En este sentido, es sensato admitir que el sistema patriarcal fang que marca las relaciones entre hombres y mujeres, es producto de un proceso de asimilación y aprendizaje de determinados comportamientos que no son aislados, ya que inciden en el comportamiento de las personas. Por consiguiente, hay que atender al hecho de que "[...] el panorama de una cultura patriarcal latente impone la disociación del hombre y la mujer para adjudicar al primero el privilegio y condición de ser sujeto, y a la mujer la condición de ser objeto" (Scott, 2005, pág. 16).

Ante esto, el sistema patriarcal fang presenta varias manifestaciones donde la mujer es construida desde la perspectiva masculina, en la que aquella acata todas las normas culturales de opresión, dominación y exclusión establecidas o pactadas únicamente por los hombres. El concepto de familia fang promueve perfectamente la socialización diferencial en función de que uno sea hombre o mujer. El equilibrio comunitario depende en gran medida que cada uno cumpla el rol que se le ha asignado. Por ello, desde que los bebés nacen, los padres se comportan de una manera distinta en función del género. Así, por ejemplo, a las niñas se las educa de una manera diferente a los niños.

La educación de las niñas es tarea de la madre, pero quien dice cómo se debe educar a esta niña es el padre quien, a su vez, se encarga de educar al niño. Uno de los principales problemas que ha acarreado este tipo de educación, es que se dé más importancia a los niños 
que a las niñas a la hora de educarlos. Si bien todos van a la escuela, existe una altísima probabilidad de que las niñas no terminen la escuela. Todo ello es fruto del modelo de socialización que se inculca desde la familia. Este modelo de socialización diferencial que trasmite los roles de género en el concepto de familia fang, ha hecho que:

Muchas veces se ha acusado a la familia tradicional, incluso a sus mujeres, de ser el principal canal de transmisión de los principios patriarcales que han regido la sociedad fang: las niñas asimilan que deben servir a los hombres, deben aprender qué cosas agradan a los hombres (PérezArmiño, 2018, pág. 55).

Es evidente que esta asimilación de la que habla Pérez Armiño, se transmite en la familia y luego se refuerza en la escuela, porque es importante también destacar que la escuela es el segundo agente de socialización donde se aumenta la socialización diferencial que se inicia en la familia. Los roles de género son asimilados desde la niñez y en base a ello, las relaciones de género se vuelven en un factor que permite que las niñas tengan una educación no igualitaria en el sentido de que se las educa para servir a los hombres, mientras que a ellos se les educa para mantener a la mujer.

Desde el punto de vista de las relaciones de género, una de las características fundamentales del concepto de familia fang, es la socialización diferencial donde "los padres fomentan la adopción de roles de género a través de las experiencias que ofrecen a sus hijos e hijas (elección de juguetes, por ejemplo)" (Monjas-Casares, 2004, págs. 11-12).

Según el planteamiento que se recoge anteriormente, los progenitores son los que fomentan la trasmisión de los roles de género. Si bien en el concepto de familia fang, la socialización de los niños y niñas no depende únicamente de los padres y madres biológicos como ocurriría en el modelo de familia occidental. Al respecto, se puede señalar que en la familia africana fang, los padres y madres, son todos aquellos que intervienen en la educación de los hijos, que pueden ser los tíos, abuelos, las tías, incluso gente de la misma tribu que no tiene un parentesco consanguíneo pero que comparte un mismo tótem con cierta familia. Sobre la cuestión en la cultura fang existen principalmente cuatro tipos de padres o madres.

En primer lugar, están los mbeiñ, se refiere a los progenitores, es decir, el padre y la madre biológicos. En segundo lugar, están los mbala, que son los padres y madres no biológicos. Pueden ser considerados como padrastros o madrastras. De hecho, en la cultura fang existe un refrán muy popular que dice así: muan ase muan mbeiñ, muan ane muan mbala. Significa que el niño no es de los progenitores, sino del que lo cuida. Esto para demostrar que no vale la pena tener descendencia si no se es capaz de cuidarla. En tercer lugar, están los esehe nvong abot, son los tios o tías de parte de la madre o del padre. En cuarto lugar, se encuentran los esehe kibot, es todo aquel que es de la edad de tu padre o madre biológico, incluso si no es de tu tribu o etnia, es tu padre o madre, por tener la edad de tus progenitores. Por eso, forman parte de la educación de los niños y niñas.

Al ser la sociedad ecuatoguineana pluriétnica, lo que se observa es que, en todas las etnias del país, ha prevalecido una cierta dominación de los hombres sobre las mujeres, debido fundamentalmente a una longeva fuerte construcción sociocultural. La presencia de la división sexual del trabajo en el concepto de familia fang a la hora de trasmitir los roles de género, evidencia sin lugar a dudas, la socialización diferencial. La división sexual del trabajo es la patente del modelo de familia fang, la cual muestra que las niñas por el mero hecho de serlo, deben realizar determinadas tareas y los niños otras. Se Los padres o las 
madres, son conscientes que los papeles o roles de género deben ser ejercidos por sus hijos, de hecho, es en casa, en la familia, donde se comienza asignar roles de género en función del sexo de la prole.

La separación de espacios, público y privado, queda totalmente establecido, de tal forma que a las niñas les corresponda siempre el espacio privado, mientras los niños asumen el espacio público. Esta manera de educar a los niños no es producto de la actualidad, sino que ya desde el comunitarismo tradicional fang se llevaba a cabo este tipo de educación promovida desde las familias. De acuerdo con eso, Nvé Bengobesama sostiene que:

Cuando los niños llegaban al uso de razón compartían la vida con los mayores en el $a b a a$, con cuya convivencia aprendían muchas cosas de la vida laboral de los hombres: coser la nipa para la construcción, tejer las nasas y cestas de pescar y preparar cualquier género de trabajo artesano (Nvé-Bengobesama, 1981, pág. 201).

Como se puede observar, el autor citado, sostiene que los niños eran los únicos que tenían acceso a ese lugar. En ningún momento menciona a la niña porque está claro que el lugar de la niña estaba en la cocina. Dentro del concepto de familia fang siempre se reserva este espacio público para los hombres, para que tomen las decisiones oportunas para con sus propias familias, convirtiéndolos de esta manera en los únicos con la capacidad de decidir y actuar sobre el destino de sus respectivas familias, los hijos y las hijas quedan exclusivamente bajo su cobertura. Así pues, la división sexual del trabajo y la asignación de los roles de género, se inculca desde el núcleo familiar.

En el concepto de familia fang, se observa que, con la asignación de los roles de género, se crea lo que es la división sexual del trabajo, la cual se manifiesta en los siguientes términos:

- Cuando las niñas son pequeñas, se las somete a la socialización primaria, que es fruto de lo que aprenden en la familia. Las madres (tías, primas, abuelas, tatarabuelas, madres de tribu, etc.), se encargan de enseñar a las niñas que, por ser mujeres, deben saber cocinar, lavar o planchar para agradar a sus maridos cuando tengan que casarse. Eso significa que es un imperativo saber cocinar, lavar, planchar y mantener limpio el hogar, para que tengas un buen esposo y ese se sienta a gusto porque de lo contrario no se casaría.

Esta manera de socializar a las niñas ha hecho, por ejemplo, que el hombre fang no le interese la cocina, porque ha aprendido que es un rol exclusivo de la mujer. Es un acto vergonzoso encontrar a un hombre en la cocina preparando la comida, porque culturalmente está mal visto debido principalmente a la manera en que, en este sentido, se ha socializado a los hombres. Es más, las mujeres asumen que es su obligación dar de comer a sus maridos e hijos. Dentro del concepto de familia fang; se ha naturalizado que las mujeres deben realizar los trabajos domésticos y los hombres deben ser proveedores de alimento a sus casas. Precisamente, el resultado de esto es que los hombres no realizan las funciones domésticas, no porque no puedan, sino porque se les ha enseñado que es el rol de las mujeres.

Por ello, "el papel (rol) de género se forma con el conjunto de normas y prescripciones que dictan la sociedad y la cultura sobre el comportamiento femenino o masculino" (Lamas, 1995, pág. 4). No es extraño que sea visto con desdén que un hombre se dedique a cocinar, porque no es un comportamiento asignado a los hombres sino a las mujeres, y la familia 
debe encargarse de que se cumpla este rol. Sin embargo, eso es un poco contradictorio porque no se puede pregonar de igualdad en unos ámbitos dejando otros.

Es importante resaltar que, para la mujer ecuatoguineana en general, es importante poseer una familia y cuidarla. De hecho, la mujer es el pilar de la familia africana, porque se encarga de la educación y cuidado de sus hijos durante la ausencia del padre, que está fuera del hogar trabajando. Muchas veces, el rol de la mujer en la educación de los hijos es tal, porque es la encargada de transmitirles los valores socioculturales, los cuales suelen ser reforzados por el padre. Cabe destacar la complementariedad que existe entre la mujer y el hombre a la hora de educar a sus hijos. Sin embargo, la educación de los niños siempre está determinada por el hombre. En otras palabras, significa que:

El hombre crea un modelo educativo, la mujer lo reproduce. Y cuando la madre se revuelve contra ese sistema de educación recibe la violencia del padre. La mujer es la que transmite, pero el poder real está en manos del hombre. Realmente ella es la que educa, pero la persona que le dice «educa así» es él [...]. En la etnia fang quien manda es el hombre. No importa lo que la mujer diga cualquier cosa. Y de hecho cuando aparece papá en casa todo el mundo se calla (Melibea-Obono, 2016).

Lo que se evidencia claramente, es que la educación de los niños y niñas, es dictada por el padre y ejecutada por la madre.

- Por otro lado, la educación de los niños es totalmente diferente a la de las niñas. Los niños en cuanto a las tareas del hogar, les enseñan hacer algunas cosas como, por ejemplo, coger agua del pozo para la casa o en algunas ocasiones, fregar la misma casa, aunque esta última tarea suele terminar siendo asumida por las niñas. La socialización diferencial de la que son producto los niños, hace que aquellos sean educados para dominar o mandar sobre la mujer.

La realidad establecida por la socialización diferencial que se fomenta dentro del concepto de familia que tienen los fang, es el resultado de su propia estructura patriarcal que sustenta todos estos comportamientos. Por consiguiente, existe de este modo una estrecha relación entre la socialización diferencial, los roles de género y la división sexual del trabajo. El punto común está en que son promovidos desde la familia que es evidentemente patriarcal.

Al considerar que la familia fang promueve un sistema de socialización diferencial, fruto de la asignación de unos roles de género a las mujeres y hombres, significa que los hombres se convierten en dominantes. Además, tampoco es un hecho aislado considerar que la división sexual del trabajo agrava considerablemente la subordinación de las mujeres, las cuales terminan siendo presentadas por el sistema patriarcal fang como inútiles, pues, aun cuando se pueda visibilizar y valorar su trabajo doméstico, es totalmente infravalorado por farios factores sobre todo culturales.

Los roles de género en el concepto de familia fang son asimétricos, así pues, "En su funcionamiento ordinario los hombres y las mujeres comparten el lecho, mientras tanto el trabajo y las actividades recreativas se llevan por separados" (Oyono Nzang, 2013, págs. 3637). La familia socializa desde la edad temprana y eso permite que cada uno vaya asumiendo su propio rol. En el caso de los hombres; tendrán a lo largo de su vida un rol activo, inculcado y aprendido desde la edad temprana, a diferencia de lo que ocurre con las mujeres, que asumen el rol pasivo y en función de eso se comportan. Si bien es cierto que se acepta que 
asistan a la escuela, lo que todavía no se está consiguiendo es que aprendan como los hombres o igual a ellos.

Los factores culturales siguen siendo ataduras para que las mujeres se sientan empoderadas, por ello, la sumisión aprendida, fruto de la socialización diferencial respecto a los roles género, marca considerablemente el comportamiento de la mujer fang. Probablemente, sea bueno repensar en un modelo de familia un poco más igualitario, puesto que anclarse a los valores culturales es sumamente correcto, siempre y cuando los mismos permitan a las personas poder actuar, crecer y vivir como tal. Todo eso porque, "la familia es el agente de socialización más importante en la vida de un individuo, no sólo porque es el primer agente, sino porque se constituye en el nexo entre el individuo y la sociedad" (Rodríguez- Pérez, 2007, pág. 92). El resultado que se tiene de ello es que cada individuo termina asumiendo y creciendo de acuerdo a como se le ha educado.

Los roles de género no se escapan del concepto de familia fang, son el motor de la desigualdad y promueven la sumisión de la mujer. Los hombres se libran del trabajo doméstico no porque no puedan hacerlo, simplemente porque este no es el rol con el que se les ha socializado. Por ello, hay que despatriarcalizar la cultura para poder construir otro modelo de socialización más igualitario. La fundamentación del sistema patriarcal fang que promueve la socialización diferencial con la asignación de los roles de género, hace que los hombres sean dominantes sobre las mujeres, porque "los roles de género fundan la base de un sistema de segregación social, personal y económica entre los sexos, el cual los hombres pertenecen a la categoría superior manteniendo el poder sobre las mujeres" (Bourdieu, 2000, págs. 37-38). Negarse a asumir que el concepto de familia fang no fomenta la socialización diferencial y por ende la asignación de roles, es pretender ocultar una realidad evidente que promueve la subordinación de la mujer.

\section{Metodología}

El propósito de este epígrafe, es presentar las técnicas, así como los instrumentos manejados en esta investigación. Para la realización de esta investigación, se ha optado primero por la metodología cuantitativa. Para ello, se ha procedido en primer lugar a una revisión sistemática de la literatura existente sobre esta temática. Es muy importante enfocarnos en esto último, pues la revisión de la literatura ha permitido, en función de los manuales y artículos académicos utilizados, argumentar sobre la socialización diferencial y la asignación de los roles de género dentro del concepto de familia fang. Hemos trabajado con las cualidades de los seres humanos que son productos de conocimiento. Además, dado que es una investigación de corte pedagógico, antropológico y sociológico, nos atrevemos afirmar que es la metodología que mejor se ajusta a esta investigación. Optar por esta metodología cualitativa ha sido notable para esta investigación, puesto que se trata de un tipo de investigación donde:

Los investigadores se interesan en comprender los significados que los individuos dan a su propia vida y a sus experiencias. El punto de vista, el sentido que los actores dan a sus conductas, o a su vida, es materia de observación y de investigación. Aquí se da valor a la subjetividad en la comprensión y la interpretación de las conductas humanas y sociales. (Anadón, 2008, págs. 198-211).

En este sentido, para consolidar nuestra investigación, se ha utilizado el método descriptivo. Un estudio descriptivo como este, “[...] se basa en una rigurosa descripción contextual de un hecho o una situación que garantice la máxima intersubjetividad en la captación de una 
realidad compleja mediante la recogida sistemática de datos que haga posible un análisis interpretativo" (Pérez, 2000, pág. 29). En otras palabras, el método descriptivo ha permitido conocer mejor la naturaleza de los roles de género en el concepto de familia fang y su sistema de socialización diferencial. En este sentido, se hace necesario un enfoque cuantitativo apoyado en el método descriptivo, y que implica que el investigador tenga un panorama general de lo que pretende conocer.

Además, también conviene señalar que para conocer mejor la cuestión que se pretende desarrollar, la técnica que se ha utilizado es la encuesta, mientras que el instrumento de recogida de datos ha sido preferentemente un cuestionario para recabar información sobre este tema. El cuestionario que se ha utilizado como instrumento de recogida de datos, es el que ha facilitado la obtención de los datos que se manejan en esta investigación, para así poder llegar a unas conclusiones. Teniendo en cuenta que esta investigación se desarrolla en Guinea Ecuatorial, la población sobre la que se ha realizado este trabajo la constituyen los fang del país mencionado. Sin embargo, a sabiendas de que los fang son un grupo étnico mayoritario en nuestra sociedad, la muestra seleccionada ha sido de 402 ciudadanos y ciudadanas que se encuentran en la ciudad de Bata. Se trata de una muestra muy representativa porque en esta ciudad se encuentran mayoritariamente representadas las tribus de la etnia fang. Por consiguiente, los datos obtenidos con esta muestra, son perfectamente generalizables a toda la población fang de Guinea Ecuatorial.

\section{Análisis y discusión de los resultados}

Los resultados que presentamos a continuación, son fruto de la encuesta realizada sobre una población muy selecta. Pensamos que estos datos, generados gracias al uso del Paquete Estadístico para las Ciencias Sociales (SPSS), permiten obtener un panorama bien fundamentado sobre los roles de género y la socialización diferencial dentro del concepto de familia fang.

\begin{tabular}{llcccc} 
& & Frecuencia & Porcentaje & $\begin{array}{l}\text { Porcentaje } \\
\text { válido }\end{array}$ & $\begin{array}{l}\text { Porcentaje } \\
\text { acumulado }\end{array}$ \\
\hline Válido & Sí & 205 & 51,0 & 51,0 & 51,0 \\
& No & 197 & 49,0 & 49,0 & 100,0 \\
& Total & 402 & 100,0 & 100,0 & \\
\hline
\end{tabular}

Cuadro 1. ¿Crees que los roles de género en el concepto de familia fang contribuyen en la subordinación de la mujer?

Según los resultados obtenidos con esta pregunta, el 51,0 \% de las personas encuestadas admitió que los roles de género contribuyen a la subordinación de la mujer fang. Sin embargo, también nos ha llamado la atención del 49,0\% que piensa que estos roles de género no son la causa de la subordinación de la mujer.

Lo que realmente se ha constatado es que la mayor parte del grupo encuestado no sabe lo que son los roles de género, sin embargo, tras explicarle que hacía referencia a los 
comportamientos, conductas que debe realizar el hombre y la mujer, comenzaron admitir que existen estos roles de género.

\begin{tabular}{llllll}
\hline & & Frecuencia & Porcentaje & $\begin{array}{l}\text { Porcentaje } \\
\text { válido }\end{array}$ & $\begin{array}{l}\text { Porcentaje } \\
\text { acumulado }\end{array}$ \\
\hline $\begin{array}{l}\text { Válid } \\
\text { o }\end{array}$ & A través de la escuela & 69 & 17,2 & 17,2 & 17,2 \\
& $\begin{array}{l}\text { A través de un sistema } \\
\text { patriarcal }\end{array}$ & 182 & 45,3 & 45,3 & 62,4 \\
& $\begin{array}{l}\text { A través de la propia } \\
\text { familia }\end{array}$ & 151 & 37,6 & 37,6 & 100,0 \\
& & & & \\
& & & & & \\
\hline
\end{tabular}

Cuadro 2. ¿Desde dónde se fomenta la socialización diferencial y los roles de género?

Los roles de género al igual que la socialización diferencial, son fruto del sistema patriarcal fang, así lo han confirmado el 45,3\% del grupo encuestado. Los roles de género son una manifestación del sistema patriarcal fang que fomenta sobre todo la socialización diferencial, teniendo como resultado, supeditar lo femenino a lo masculino.

Todo ello genera de esta manera unos comportamientos que se ven como normales, pero que en realidad son producto de aquello que se ha enseñado desde la familia. También es importante destacar el que 37,6 \% de las personas encuestadas cree que la familia es el germen de los roles de género y de la socialización diferencial.

\begin{tabular}{llllll}
\hline & & Frecuencia & Porcentaje & $\begin{array}{l}\text { Porcentaje } \\
\text { válido }\end{array}$ & $\begin{array}{l}\text { Porcentaje } \\
\text { acumulado }\end{array}$ \\
\hline Válido & Sí & 205 & 51,0 & 51,0 & 51,0 \\
& No & 197 & 49,0 & 49,0 & 100,0 \\
& Total & 402 & 100,0 & 100,0 & \\
\hline
\end{tabular}

Cuadro 3. ¿Crees que la socialización diferencial limita el desarrollo personal de la mujer?

Si algo hemos evidenciado a lo largo de esta investigación es que dentro del concepto de familia fang, los niños y las niñas son socializados de manera diferente. Así pues, el 51,0\% de las personas encuestadas considera que estos roles y la manera de socializar a la gente dentro de concepto de familia fang, limita el desarrollo personal de la mujer, por confinarla 
al ámbito de lo privado, donde se dedica a las tareas domésticas y al cuidado de los niños y las niñas. Frente a esta posición, existe un 49,0\% que rechazan que la socialización diferencial limite a la mujer.

\begin{tabular}{|c|c|c|c|c|c|}
\hline & & Frecuencia & Porcentaje & $\begin{array}{c}\text { Porcentaje } \\
\text { válido }\end{array}$ & $\begin{array}{l}\text { Porcentaje } \\
\text { acumulado }\end{array}$ \\
\hline \multirow[t]{3}{*}{ Válido } & Sí & 321 & 79,9 & 79,9 & 79,9 \\
\hline & No & 81 & 20,1 & 20,1 & 100,0 \\
\hline & Total & 402 & 100,0 & 100,0 & \\
\hline
\end{tabular}

Cuadro 4. ¿Los roles de género, productos de la socialización diferencial fomentan la división sexual del trabajo en el concepto de familia fang?

Para el 79,9\% de las personas encuestadas, los roles de género fomentan la división sexual del trabajo, algunos han afirmado eso porque ven que deben hacer ciertas tareas que se les ha asignado. Así, por ejemplo, las mujeres se dedican a planchar o cocinar, mientras que los hombres simplemente están buscando sustento para la familia. El resultado de esta socialización diferencial es, como se ha visto anteriormente, la reclusión de la mujer al ámbito privado que representa el hogar. Esto se debe al sistema patriarcal fang que, con esta manera de socializar, crea una sociedad diferencial de la que no es ajena a la familia fang. Además, pese a existir un 20,1\% que piensan que los roles de género no fomentan la división sexual del trabajo, probablemente sea porque no saben realmente lo que son los roles de género, pues son temas de reciente incorporación en el estudio de la realidad social de la sociedad ecuatoguineana desde el punto de vista de las relaciones de género.

\section{Conclusión}

El sistema de socialización diferencial que se observa en el concepto de familia fang, fomenta la transmisión de los roles de género tradicionales, donde el hombre por lo general es educado como un ser dominante, mientras que la mujer debe mostrar obediencia, sumisión y debilidad. Si bien la familia es una de las grandes instituciones de la etnia fang, es la principal transmisora de los roles de dependencia que asume la mujer en su seno. En este sentido, esta investigación ha partido de la tesis de que el concepto de familia fang promueve la socialización diferencial a través de la transmisión de los roles de género que convierten a la mujer en una sumisa, robusteciendo el sistema patriarcal sobre el que se sustenta este modelo de socialización, con el propósito de analizar y entender mejor una temática que cuenta con escasísima literatura, pues son cuestiones sociales de actualidad que hasta ahora no se habían planteado a la luz de las relaciones de género, partiendo del componente de la socialización diferencial.

La tesis presentada se ha argumentado en base a algunas evidencias como es la presencia de la división sexual del trabajo que es la manifestación del sistema patriarcal que crea los roles de género y que al mismo tiempo son sustentados por el sistema patriarcal fang. Por mucha apología que se quiera hacer del modelo de familia fang, que representa al mismo tiempo la concepción de la familia africana, hay algo ineludible, y es la presencia de los roles de género que se fomentan con la socialización diferencial que se lleva a cabo desde la familia. Por consiguiente, es un hecho evidente que en el concepto de familia fang se lleva a cabo una segmentación entre hombres y mujeres, la cual es reforzada en la escuela, 
provocando así las desigualdades sociales instauradas desde la primera socialización, lo cual conduce inexorablemente a la diferenciación de los roles y espacios.

Una sociedad donde se lleva a cabo este tipo de socialización que favorece a un género infravalorando al otro, preferentemente femenino, institucionaliza el patriarcado. Es más, le rinde pleitesía por no promover modelos de educación inclusivos para todos y todas. Ante esto, conviene ser contundentes por abolir este sistema de segregación, el cual se puede combatir exclusivamente con la educación, pero debe ser una educación igualitaria.

Mientras que con la división sexual del trabajo las niñas siguen siendo educadas o socializadas para dedicarse únicamente al cuidado de sus hijos e hijas y complacer a los hombres, será difícil doblegar el sistema patriarcal, por eso, la solución es cambiar el modelo de socialización diferencial actual para dar paso a uno más comprometido por explotar y valorar las aptitudes y capacidades de las personas que no dependen del género de uno.

Con el sistema de socialización diferencial actual del modelo de familia fang donde todos educan (padres, tíos, primos, hermanos biológicos, hermanos de la tribu, los abuelos, etc.), se agravan los roles de género, los cuales hacen que las niñas sean socializadas para desarrollar conductas como la dependencia y la sumisión, reprimiendo su capacidad cognitiva porque quedan confinadas al ámbito de lo privado donde se dedican exclusivamente a la crianza de los hijos e hijas y de servir a los hombres.

Al ser los roles de género una construcción sociocultural presente en el concepto de familia fang, nos interpela a tener que estudiar la manera en la que fomentan la desigualdad social, desde la socialización diferencial. Por eso, es relevante llevar a cabo una serie de estudios que tengan el propósito de visibilizar estas situaciones para promover cambios que pretendan instaurar un sistema más igualitario entre los sexos. La educación y el fomento de las oportunidades son una exigencia ética y moral para el desarrollo de las capacidades de las personas sin importar su sexo y género. Aferrarse a las costumbres y tradiciones no debe implicar infravalorar a nadie, sino más bien fomentar el desarrollo de la persona humana. 


\section{Bibliografía}

Ki-Zerbo, J. (1972). Historia de África Negra 2 Vols. Lisboa: Europa-América.

Mbiti, J. (1990). Entre Dios y el tiempo. Religiones tradicionales Africanas. Madrid: Mundo Negro.

Musitu, G., \& Cava, M. J. (2001). La familia y la educación. Barcelona: Ediciones Octaedro, S.L.

Meil Landwerlin, G. (2006). Padres e hijos en la España actual. Barcelona: Edita Fundación La Caixa.

Papalia, D. (2005). Psicología del desarrollo. México: McGraw-Hill.

Maurier, H. (1966). "Religión y desarrollo: Tradiciones Africanas y Catequesis". Cuadernos Mundo Negro 4.

Castells, M. (1998 ). La era de la información. México: Siglo XXI.

Scott, C. E. (2005). Sobre Los lazos de amor. Psicoanálisis, feminismo y el problema de la dominación de Jéssica Benjamín. Gaceta Universitaria: Temas y controversias en Psiquiatría, pp. 16-19.

Mibui, R. E. (2005). Historia de Guinea Ecuatorial: período pre-colonial. Madrid: Gráficas Algoran.

Lamas, M. (1995). La perspectiva de género. Revista de Educación y Cultura de la sección 47 del SNTE, pp.14-20.

Oyono Nzang, J. E. (2013). El hombre en busca de su Dios. Amigo del Hogar.

Bourdieu, P. (2000). La dominación masculina. Barcelona: Anagrama.

Anadón, M. (2008). La investigación llamada "cualitativa": de la dinámica de su evolución a los innegables logros y los cuestionamientos presentes. Investigación y Educación en Enfermería, XXVI(2), 198-211.

Pérez, G. (2000). Investigación cualitativa, retos e interrogantes II, técnicas y análisis de datos. España: Editorial la Muralla.

Myers, D. G. (2006). Psicología. Buenos Aires: Médica Panamericana.

Ares-Muzio, P. (2002). Psicología de la Familia. Una aproximación a su estudio. La Habana: Féux Várela.

Nvé-Bengobesama, C. O. (1981). Tradiciones del pueblo fang. Madrid: RIALP, S.A.

Monjas-Casares, M. I. (2004). Ni sumisas ni dominantes: Los estilos de relación interpersonal en la infancia y la adolescencia. España: Ministerio de Trabajo y asuntos Sociales.

Pérez-Armiño, L. (2018). "Y el sujeto se hizo verbo (aunque siempre fue objeto). La mujer fang en Guinea Ecuatorial y el impacto colonial". Anales del Museo Nacional de Antropología, XX. Madrid: Ministerio de Educación, Cultura y Deporte, pp. 30-59.

Nzé-Abuy, R. M. (1985). Familia y matrimonio fán. Madrid: Anzós S. A.

Santelices-Cuevas, L. (2001). La familia desde una mirada antropológica: Requisito para educar. Pensamiento Educativo. Vol. 28, pp.183-198. 
Melibea-Obono, T. (15 de Diciembre de 2016). "En España me llaman "la negra", en Guinea Ecuatorial "la españolita". Obtenido de Recuperado el 10 de julio de 2020, de https://www.abc.es/cultura/cultural/abci-trifonia-melibea-obono-espanallaman-negra-guinea-ecuatorial-espanolita-201612121313_noticia.html

Martínez- González, R.-A. (1996). Familia y Educación. Oviedo: Servicios de Publicaciones Universidad de Oviedo.

Rodríguez- Pérez, A. (2007). Principales modelos de socialización familia. Foro de educación no 9, pp. 91-97. 


\section{Autor}

PEDRO BAYEME-BITUGA obtuvo en 2017 su título de Graduado en Humanidades, en el perfil de cooperación internacional y Desarrollo por la Facultad de Humanidades y Ciencias Religiosas de la Universidad Nacional de Guinea Ecuatorial. En 2020 obtuvo el título de Máster Universitario en estudios Humanísticos y Sociales de la Universitat Abat Oliba-CEU de Barcelona. Es doctorando en Humanidades para el Mundo Contemporáneo, en la línea de investigación de Historia y Sociedad, del programa de doctorado de la Escuela Internacional de Doctorado CEU (Universitat Abat Oliba-CEU de Barcelona).

Actualmente es profesor titular de la Facultad de Humanidades y Ciencias Religiosas de la Universidad Nacional de Guinea Ecuatorial donde imparte las asignaturas de Sexualidad, Género y Poder, y de Metodología de investigación científica. Sus principales temas de investigación incluyen las cuestiones feministas y culturales de su contexto. Además, es miembro cofundador del grupo pódium humanitas. Es autor de algunos artículos publicados en revistas de gran calibre científico como la Revista Cátedra, Academia.Edu, etc. Ha dirigido varios Trabajos Fin de Grado relacionados con sus líneas de investigación. 